\title{
Antimicrobial susceptibility pattern, and associated factors of Salmonella and Shigella infections among under five children in Arba Minch, South Ethiopia
}

Gemechu Ameya ${ }^{* *} \mathbb{0}$, Tsegaye Tsalla ${ }^{1}$, Fasil Getu² and Eyob Getu ${ }^{3}$

\begin{abstract}
Background: Diarrheal diseases continue to be the major cause of morbidity and mortality among children under 5 years. Salmonella and Shigella specious are the major enteric pathogen causing diarrhea among children worldwide. Examination of stool sample is the most sensitive method to diagnose diarrheal disease in children. This study aimed to determining the prevalence, antimicrobial susceptibility pattern and associated factor of Salmonella and Shigella infection among under five children.
\end{abstract}

Methods: A cross sectional study was conducted on under 5 years children attending Arba Minch town. Pre-tested and structured questionnaire was used for collecting data about socio-demographic characteristics and associated factors. Stool sample was used to isolate and identified the pathogen. Antimicrobial susceptibility test was performed for isolated Salmonella and Shigella specious. A logistic regression analysis was used to see the association between different variables and outcome variable. Odds ratio with $95 \% \mathrm{Cl}$ was computed to determine the presence and strength of the association.

Results: A total of 167 under five children were included in the study. About $57 \%$ of participants were males with the mean age of 32 months. The overall prevalence of Salmonella and Shigella species infection was 17.45\% with $12.6 \%$ Salmonella species. The isolates were resistant to common antibiotics such as Amoxicillin, Erythromycin, Chloramphenicol, Clindamycin, Norfloxacin, Ciprofloxacin, Cotrimoxazole, and Gentamycin. Urban resident [AOR $=7.11$; $95 \% \mathrm{Cl}(2.3,22.2)]$, month income < 1000 Ethiopian birr $[\mathrm{AOR}=6.5 ; 95 \% \mathrm{Cl}(2.0,21.4)]$, absence of waste disposal system $[A O R=3.3 ; 95 \% \mathrm{Cl}(1.2,9.3)]$, poor hand washing habit $[A O R=6.0 ; 95 \% \mathrm{Cl}(2.0,18.2)]$, untrimmed finger nail $[A O R=3.7 ; 95 \% \mathrm{Cl}(1.4,10.6)]$, and use of napkin $[A O R=3.2 ; 95 \% \mathrm{Cl}(1.1,9.3)]$ had significant association with Salmonella and Shigella infection.

Conclusion: Salmonella and Shigella species infections were higher as compared the national prevalence. This study also revealed that the enteric infection were significantly associated with finger nail status, residence, hand washing practice, month income of parents, usage of napkin after toilet, and absence of waste disposal system. Therefore, working on identified associated factors and regular drug susceptibility test is mandatory to reduce the problem.

Keywords: Antimicrobial activity, Diarrhea, Salmonella, Shigella, Under five children

\section{Background}

\footnotetext{
*Correspondence: gemechuameya@gmail.com

1 Department of Medical Laboratory Science, College of Medicine and Health Sciences, Arba Minch University, P.O. Box: 21, Arba Minch Ethiopia

Full list of author information is available at the end of the article
}

Diarrhea is a worldwide problem that frequently encountered in the practice of pediatric medicine. Globally 550 million people annually become ill. Of these 220 million of cases occur in children under the age of 5 years [1]. According to the world health organization report diarrheal illness is also the second leading causes of death in 
children younger than 5 years. Globally $21 \%$ of deaths in children under the age of 5 years results from diarrheal infection [2]. Diarrhea kills more young children than Malaria, AIDS, and Measles combined [3]. In developing countries, Shigella and Salmonella species remain major contributors to acute enteric infection in children. Asia, Africa and Latin America had an estimated 2.5 million deaths each year in children under the age of five [4]. In persist cases it can cause dehydration due to large amount of fluid loss which often begin with loss of the normal stretchiness of the skin and irritable behavior. Further complication may result in decreased urination, loss of skin color, and fast heart rate $[5,6]$.

Diarrhea is mainly caused by infection with virus, bacteria or parasite [4]. Diarrhea caused by parasite has slow onset of action unlike that of bacterial or viral infection. Under normal condition, the gastrointestinal tract has great capacity to absorb fluid and electrolyte. However, enteric pathogens disturb this balance by different mechanisms. All this action results in fluid and electrolyte loss and this may leads to death [7]. Bacterial diarrhea is commonly caused by Salmonella enterica, Shigella species, Vibrio cholera, Clostridium difficile, Escherichia coli, Campylobacter jejuni and others. Among these Salmonella and Shigella specious are the endemic in most part of developing countries [4].

Salmonella causes Salmonellosis which can be characterized by diarrhea, fever, vomiting and abdominal cramps after $12-72 \mathrm{~h}$ of infection. Salmonella enteric serotype typhi is the common serotype of Salmonella that causes of typhoid fever. Typhoid fever is a systemic disease with diarrhea and it is the major causes of morbidity and mortality worldwide in under the age of five children [8]. Shigellosis is also known as bacillary dysentery or Marlow syndrome is caused by Shigella species and it can rarely occur in animals other than humans [9]. In addition Shigella dysenteriae species releases Shiga toxin, an AB exotoxin similar to Entero EHEC that cause diarrhea [10].

Different factors like socio-economic characteristic of the patient, poor access to latrine, lack of clean drinking water can be associated with diarrhea diseases. Poverty is a good indicator of acute children diarrhea. It is associated with poor housing, crowding, dirty floor, lack of clean water and poor food storage condition. It can also be associated with cohabitation with domestic animals [11]. Laboratory diagnosis of Salmonella and Shigella are mainly performed by culturing of the organism or through the demonstration of specific antibody or antigen in the serum or urine. Now a days, multiple drug resistant Shigella and Salmonella species are frequently isolated in clinical samples. This makes them difficult to be treated. Antimicrobial resistance within wide range of infectious agents is growing public health treat of the twenty-first century [12].

Nowadays different researches conducted in Ethiopia showed that multiple drug resistant Salmonella and Shigella species are isolated from children under the age of five [13]. There is limited published research on prevalence, antimicrobial susceptibility pattern and associated factor of Salmonella and Shigella infection among under the age of five children in southern part of Ethiopia. Therefore, this study is aimed to determine the prevalence, drug sensitivity pattern and associated factors of Shigella and Salmonella species infections among under five children in Arba Minch town Governmental Health Institutions, southern Ethiopia.

\section{Materials}

\section{Study design, setting, and period}

An institutional based cross sectional study was conducted on governmental health institute on antimicrobial sensitivity and factor associated with Salmonella and Shigella infections among under the age of five children. The study was conducted in Arba Minch town governmental health institutes, southern part of Ethiopia. The study was conducted from March to May, 2017.

\section{Source population and sampling process}

Under five children attending at the three governmental health institutions of Arba Minch town (Arba Minch General Hospital, Shecha Health Center, and Sikela Health Center) during study period were source population. Systematic sampling method was used to select 167 under five children with diarrhea disease who fulfill inclusion criteria during the study period using case interval. The first case of the interval was selected by lottery method. All under five children with diarrhea disease were included in the study. Patients who had received antibiotic treatment within 1 week for their symptom before coming to hospital were excluded from the study.

\section{Data collection}

A pre-tested, structured questionnaire was used to collect socio-demographic, socio-economic and clinical data of the children. The samples were proportionally allocated in each health institutions. Information related to children age, sex, family income, family size, educational status of the parent/guardian, family occupation, latrine usage, hand washing habit before having meal, hand washing habit after visiting toilet, hand washing habit after changing napkins, availability of dry and liquid waste disposal system, feeding practice, vegetable washing habit, and finger nail status. 


\section{Stool sample collection}

Parent or care giver of children was informed to bring stool sample of their children. A clean, dry and leak proof stool cup and clean wooden applicator stick were given for stool specimen collection. The children attendant was informed to bring approximately $2 \mathrm{~g}$ of stool. After collection the stool sample was transported to Arba Minch University College of Medicine and Health Science, Microbiology Laboratory within $1 \mathrm{~h}$.

\section{Stool examination}

First physical examination of the stool sample was performed in order to check whether the sample was diarrheal or not, presence of blood, pus and mucus. Immediately after physical examination of fecal specimen, direct microscopic examination was performed. This involves reporting of the appearance of the specimen and identifying any parasitic larva, ova, cyst, and trophozoite of some parasites microscopically.

\section{Culturing and identification}

The collected stool specimen was processed for bacteriological analysis. The stool sample was inoculated into MacConkey (Oxoid Ltd.) and Xylose Lysine Deoxycholate agar (Oxoid Ltd.) by using sterile wire loop. The inoculum was incubated under aerobic condition at $37^{\circ} \mathrm{C}$ for $24 \mathrm{~h}$. After period of incubation, the plates were examined for colony characteristics of Salmonella and Shigella specious. Colorless to yellow colonies on MacConkey agar, and Pink to red colonies on Xylose lysine deoxycholate agar were further identification of species of pure isolates by biochemical tests [14].

\section{Antimicrobial susceptibility testing}

In vitro antimicrobial susceptibility test was carried out for identified Salmonella and Shigella species. The susceptibility test was performed on Muller Hinton agar by using Kirby-Bauer disc diffusion technique. Precisely, pure identified colonies from the overnight culture was suspended in nutrient broth and incubated for $4 \mathrm{~h}$ at $37{ }^{\circ} \mathrm{C}$. Turbidity of broth culture was checked against 0.5 McFarland standards. By using sterile swab the organism in broth was uniformly inoculated into Muller Hinton agar. The antibiotic discs were applied on the surface of the inoculated agar. Antimicrobial disc were selected according to committee for clinical laboratory standard (CCLS), 2015 list of drug for specific isolated pathogen and based on locally availability of the drug. The drugs used in the antibiotic susceptibility test are Amoxicillin, Erythromycin, Chloramphenicol, Clindamycin, Norfloxacin, Ciprofloxacin, Cotrimoxazole, and Gentamycin. After overnight incubation, the diameter of growth inhibition around the discs was measured and interpreted as sensitive, intermediate or resistant according to clinical and laboratory standards institute (CLSI) [15]. Known Salmonella and Shigella species were used as positive control in all methods.

\section{Data processing}

Collected data was entered to Epi-Info version 7.2.1 and exported to SPSS version 20.0 for further analysis. Descriptive statistics and logistic regression were used for analysis of data. Logistic regression analysis was used to look into the association between associated factors and bacterial enteric infection. Odds ratio (OR) and $95 \%$ confidence interval (CI) were used to determine significance between Salmonella and Shigella specious and different variables. Whose variables with level statistically significant $\mathrm{P}<0.25$ on Bivariate analysis were entered jointly into a multivariate logistic regression. $P$ value less than 0.05 was considered as statistically significant.

\section{Results}

Socio-demographic characteristics

A total of 167 children with diarrhea were included in our study in which study participants were proportionally allocated for the three Arba Minch governmental health institutes particularly Arba Minch general hospital, Sikela health center and Secha health center. The age of participants range between 5 and 59 months and mean age was 32 months. Of all the study participants, $43.1 \%$ were females and nearly half of the participants were urban dweller. About one-fourth of caregivers or parents of the children were illiterate while one-third of them learned primary education. One-fourth of the children were single parent. Among all caregivers' or parents' of the children $33 \%$ were housewife (Table 1).

\section{Magnitude of enteric pathogen}

Of 167 diarrheic children, Salmonella species was detected in $12.6 \%$ [95\% CI (8.6-16.60)] study participants and Shigella species was detected in 4.8\% [95\% CI (2.1-7.5)] children. About one-third of detected Salmonella cases were in infants and Shigella species were more detected in age group between 3 and 5 years. Among 29 exclusive breast feeding children, Salmonella species was isolated in $5(17.2 \%)$ of them and one Shigella case was also observed. Urban dwellers were more infected with the enteric pathogen than rural resident. Among children of illiterate parents, the magnitude of the enteric pathogens was one-fifth the children.

\section{Antimicrobial susceptibility test}

All isolated Salmonella species were not sensitive for Ampicillin and Erythromycin. The isolated enteric 
Table 1 Socio-demographic status of study participants

\begin{tabular}{|c|c|c|}
\hline Variables & Frequency & Percent \\
\hline \multicolumn{3}{|l|}{ Sex } \\
\hline Male & 95 & 56.9 \\
\hline Female & 72 & 43.1 \\
\hline \multicolumn{3}{|l|}{ Age (in year) } \\
\hline$<1$ & 33 & 19.8 \\
\hline $1-3$ & 65 & 38.9 \\
\hline $3-5$ & 69 & 41.3 \\
\hline \multicolumn{3}{|l|}{ Resident } \\
\hline Urban & 80 & 47.9 \\
\hline Rural & 87 & 52.1 \\
\hline \multicolumn{3}{|c|}{ Educational status of attendants } \\
\hline Illiterate & 45 & 26.9 \\
\hline Primary education & 55 & 32.9 \\
\hline Secondary education & 41 & 24.6 \\
\hline College and above & 26 & 15.6 \\
\hline \multicolumn{3}{|l|}{ Marital status } \\
\hline Unmarried & 19 & 11.4 \\
\hline Married & 126 & 75.4 \\
\hline Divorced & 16 & 9.6 \\
\hline Widow & 6 & 3.6 \\
\hline \multicolumn{3}{|c|}{ Monthly income (Ethiopia Birr) } \\
\hline$<500$ & 36 & 21.6 \\
\hline $500-1000$ & 47 & 28.1 \\
\hline $1000-1500$ & 47 & 28.1 \\
\hline$>1500$ & 37 & 22.2 \\
\hline \multicolumn{3}{|l|}{ Family size } \\
\hline$<3$ & 29 & 17.4 \\
\hline $3-5$ & 64 & 38.3 \\
\hline$>5$ & 74 & 44.3 \\
\hline \multicolumn{3}{|l|}{ Occupation } \\
\hline Housewife & 56 & 33.5 \\
\hline Private & 52 & 31.1 \\
\hline Government employer & 36 & 21.6 \\
\hline Student & 17 & 10.2 \\
\hline Other & 6 & 3.6 \\
\hline
\end{tabular}

pathogens have resistant rate of $48,44.8$, and $34.5 \%$ against Clindamycin, Chloramphenicol, and Cotrimoxazole, respectively. Ciprofloxacin was sensitive for majority (89.65) of the isolated enteric pathogen. Norfloxacin and Gentamycin has sensitivity rate of 57.38 and $52.4 \%$ against Salmonella isolates, respectively (Table 2).

Resistance for one or more antibiotics was observed among the isolated enteric pathogens. Nearly $60 \%$ of isolated pathogens were resistant to two drugs (Ampicillin and Erythromycin). About third of isolated Salmonella and Shigella species were multi drug resistant. High resistances were observed for Ampicillin and Erythromycin. The resistant rate both species for Ampicillin,
Erythromycin and Chloramphenicol was 37.5. The pathogen resistant to four and five drugs were 20.68 and $10.34 \%$ of the total isolates, respectively (Fig. 1).

\section{Factors associated with enteric pathogenic infection}

Logistic regression analysis was performed to check whether each independent predictor had significant association with the outcome variable after checking the fitness of the model for the variable by Hosmer and Lemeshow. Those predictors that were identified by bivariate analysis were adjusted by using multivariable backward stepwise logistic regression. Binary logistic regression of the socio-demographic, medical condition, and associated factors variables showed that age, marital status, residence, monthly income, occupation, finger nail length, hand washing habit, availability of latrine, usage of napkin, and availability of west disposable site were transferred into multivariable logistic regression.

Among variables transferred into multivariable logistic regression, finger nail trimming status, residence, hand washing habit, availability of waste disposal system, month income, and usage of napkins had significant association with Salmonella and Shigella infection of under five children. Those children who live in urban have about seven times more chance of infection with Salmonella or Shigella than those children who live in rural area $[\mathrm{AOR}=7.11 ; 95 \%$ CI $(2.3,22.2)]$. Parent with month income less than 1000 Ethiopian birr have about 6.5 odd of infection of their under five children with the enteric pathogen than parent with more than 1000 birr $[\mathrm{AOR}=6.5$; $95 \%$ CI $(2.0,21.4)$ ]. Children who live in environment without waste disposal system have about three times chance of infection with Salmonella/Shigella than those children from proper west disposal environment $[\mathrm{AOR}=3.3$; $95 \%$ CI $(1.2,9.3)]$. Absence of proper hand washing habit have six times odds of under five children Salmonella/ Shigella infection than those who have hand washing habit before meal $[\mathrm{AOR}=6.0 ; 95 \%$ CI $(2.0,18.2)]$. Children with untrimmed finger nail have about four times chance of getting the enteric infection than children with trimmed finger nail [AOR $=3.7 ; 95 \%$ CI (1.4, 10.6)]. Usage of napkin reduces chance of Salmonella/ Shigella infection of under five children by three times $[\mathrm{AOR}=3.2$; 95\% CI $(1.1,9.3)]$ (Table 3$)$.

\section{Discussion}

In this study a total of $29(17.45 \%)$ positive cultures for Salmonella/Shigella infection from 167 patients were identified. Among the positive cultures, Salmonella was detected in $12.6 \%$ and Shigella was detected in $4.8 \%$. This finding is similar to studies conducted in Jimma [16], Addis Ababa [13] and Hawassa [17]. In addition to this, 
Table 2 Antimicrobial susceptibility patterns of Salmonella and Shigella isolates in under five children in Arba Minch town Governmental Health Institutions

\begin{tabular}{|c|c|c|c|c|c|c|c|c|c|}
\hline \multirow[t]{2}{*}{ Antibiotics } & \multicolumn{3}{|c|}{ Salmonella species no (\%) } & \multicolumn{3}{|c|}{ Shigella species no (\%) } & \multicolumn{3}{|c|}{ Total no (\%) } \\
\hline & $\mathrm{S}$ & I & $\mathbf{R}$ & $S$ & 1 & $\mathbf{R}$ & $S$ & I & $\mathbf{R}$ \\
\hline AMP & 0 & 0 & $21(100)$ & 0 & 0 & $8(100)$ & 0 & 0 & $29(100)$ \\
\hline ERY & 0 & $12(57)$ & $9(43)$ & 0 & $3(37.5)$ & $5(62.5)$ & 0 & $15(52)$ & $14(48)$ \\
\hline CAM & $5(24)$ & $7(33)$ & $9(43)$ & $1(12.5)$ & $3(37.5)$ & $4(50)$ & $6(20.7$ & $10(34.5)$ & $13(44.8)$ \\
\hline CLI & $9(42.8)$ & $7(33)$ & $5(24)$ & $1(12.5)$ & $4(50)$ & $3(37.5)$ & $1(3.5)$ & $11(38)$ & $17(58.5)$ \\
\hline NX & $12(57)$ & $8(38)$ & $1(4.8)$ & $4(50)$ & $3(37.5)$ & $1(12.5)$ & $13(44.8)$ & $11(38)$ & $5(17.2)$ \\
\hline CIP & $20(95.24)$ & $1(4.76)$ & 0 & $6(75)$ & $2(25)$ & 0 & $26(89.65)$ & $3(10.34)$ & 0 \\
\hline CTX & $7(33)$ & $6(29)$ & $8(38)$ & $3(37.5)$ & $3(37.5)$ & $2(25)$ & $10(34.5)$ & $9(31)$ & $10(34.5)$ \\
\hline GEN & $11(52.4)$ & $6(29)$ & $4(19)$ & $3(37.5)$ & $3(37.5)$ & $2(25)$ & $14(48)$ & $9(31)$ & $6(21)$ \\
\hline
\end{tabular}

$S$ sensitive, $R$ resistant, / intermediate, AMP Amoxicillin, ERY Erythromycin, CAM Chloramphenicol, CLI Clindamycin, NX Norfloxacin, CIP Ciprofloxacin, CTX Cotrimoxazole, GEN Gentamycin

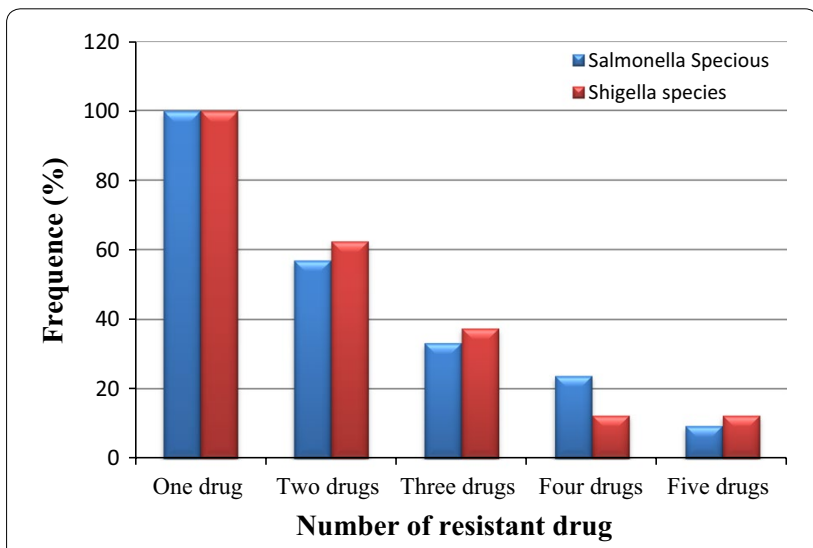

Fig. 1 Multi drug resistance pattern of bacterial isolates. [One drug (AMP), two drugs (AMP and ERY), three drugs (AMP, ERY, and CAM), four drugs (AMP, ERY, CAM, and CLI), five drugs (AMP, ERY, CAM, CLI, and NX)]

the result of this study was in line with the study conducted in Nepal [18], Turkey [19] and Nairobi [20] where $4.6,3.2$, and $2.5 \%$, respectively. Our study has lower prevalence of Shigella compared to the study conducted in Bahir Dar [21], Mekelle [22], Anyigba [23] and Gaborone [24] with prevalence of $7.85,13.3,21.9$ and $21 \%$ respectively. In contrast, the study conducted in Nigeria-Benin Teaching Hospital, is lower than our finding (1.4\%) [25]. The variation may be due to the difference in sociodemographic status, altitude, water supply or population size of the two cities.

The magnitude of Salmonella species was $12.6 \%$ in the under five children with diarrhea. The finding of this study is similar to the finding of Kenya-Igembe district hospital which found to be $10.4 \%$ [26]. A higher prevalence was observed when it is compared with the study conducted in Jimma [16] and Bahir Dar [21] in which prevalence was 6.2 and $7.8 \%$, respectively. However, this result showed an increase in prevalence of Salmonella when compared with the result of Addis Ababa [13], Hawassa [17], Turkey [19], and Kenya [20] with 3.95, 1.5, 3 and $2.5 \%$, respectively. The variation may be due to the socio-economic status, source of drinking water supply, sanitation and hygiene practice of the people in the cites.

In this study the prevalence of Salmonella and Shigella was higher in children between the age group of 1-3. The possible reason may be due to less immune status against the enteric pathogen and lack of effective follow up from their parents/caregiver. About one-third of infected children were from illiterate family. This shows that, most illiterate families didn't provide good health care for their child than those who educated. In our study the magnitude of enteric pathogens were high in children who feed breast milk alone. This may be due to less number of normal floras that help to prevent pathogens when compared to children who start complementary food. Therefore, the variations may be due to the starting of children to self-feeding that help to develop microbial flora.

This study revealed that factors associated such as residence of the children, month income of the parent, availability of west disposal system of the children living environment, hand washing habit, finger nail status, and usage of napkin associated with Salmonella and Shigella infection among the under five children. Under five children who live in urban area at risk of the enteric pathogen infection This may be due large number of population in urban area and in association with this there is large production of wastes. In our study children came from area were there was no waste disposal system had three times more chance of infection with Salmonella/Shigella infection than those came from area where there is waste disposal system. Studies also showed association between domestic wastes and enteric pathogen infection [27, 28]. 
Table 3 Factors associated with Salmonella and Shigella infections among under 5 years children in Arba Minch Governmental Health Institutions

\begin{tabular}{|c|c|c|c|c|c|}
\hline Variable & Salmonella/Shigella infection & No Salmonella/Shigella & COR $(95 \% \mathrm{Cl})$ & AOR $(95 \% \mathrm{Cl})$ & P-value \\
\hline \multicolumn{6}{|l|}{ Residence } \\
\hline Urban & 21 & 59 & $3.5(1.4,8.5)$ & $7.11(2.3,22.2)$ & 0.001 \\
\hline Rural & 8 & 79 & 1 & 1 & \\
\hline \multicolumn{6}{|c|}{ Month income (ET Birr) } \\
\hline$<1000$ & 23 & 60 & $4.9(1.9,13.0)$ & $6.5(2.0,21.4)$ & 0.002 \\
\hline$>1000$ & 6 & 78 & 1 & 1 & \\
\hline \multicolumn{6}{|c|}{ Availability of waste disposal system } \\
\hline Available & 11 & 87 & 1 & 1 & \\
\hline Not available & 18 & 51 & $2.8(1.22,6.4)$ & $3.3(1.2,9.2)$ & 0.023 \\
\hline \multicolumn{6}{|c|}{ Hand washing habit } \\
\hline Yes & 7 & 83 & 1 & 1 & \\
\hline No & 22 & 55 & $4.7(1.9,11.8)$ & $6.0(2.0,18.2)$ & 0.001 \\
\hline \multicolumn{6}{|l|}{ Finger nail } \\
\hline Trimmed & 10 & 93 & 1 & 1 & \\
\hline Untrimmed & 19 & 45 & $3.9(1.7,9.1)$ & $3.7(1.4,10.6)$ & 0.011 \\
\hline \multicolumn{6}{|c|}{ Usage of Napkin } \\
\hline Yes & 18 & 57 & $2.3(1.02,5.3)$ & $3.2(1.1,9.3)$ & 0.03 \\
\hline No & 11 & 81 & 1 & 1 & \\
\hline
\end{tabular}

Under five children who had untrimmed finger nail have about four times more odds of Salmonella and Shigella infection than those who had trimmed finger nail. Similarly under five poor hand washing habit of parent/ caregiver or children had about four times more odds of Salmonella and Shigella species infection than those who wash their hand before meal. This is in line with the study conducted Igembe district hospital, Kenya which determined hand washing practice before meal as a factor for Salmonella and Shigella species infections [26]. Unlike this study, a study conducted in Jigjiga, Somali region, Ethiopia revealed that major factors exposing to diarrheal infection were education of the primary caretaker, occupations of the father [29]. The variations may be due to difference in culture, the way of life and socio-demographic characteristics of between the study groups.

Antimicrobial susceptibility test was performed for isolated Salmonella and Shigella species revealed that both enteric pathogens were not sensitive for Ampicillin. About $42 \%$ of Salmonella and 62.5 of Shigella were resistant to Erythromycin. This study is line with the study conducted in Hawassa which revealed that majority of the isolated Salmonella and Shigella showed resistant to Erythromycin [17]. The isolated enteric pathogens were resistant to Chloramphenicol, Cotrimoxazole and Clindamycin with 43, 38 and $24 \%$ magnitude respectively. This result is similar to the study conducted in Jimma [16]. Majority of Salmonella and Shigella species were sensitive Ciprofloxacin. This result is in line with the study conducted in Bahir Dar, North Ethiopia [21]. Multi drug resistance was observed in majority of the isolated Salmonella and Shigella. Similar to this study multi drug resistant strains were observed in the study conducted in Addis Ababa [13], Nepal [18], and Bahir Dar [21].

\section{Conclusion}

The magnitude of Salmonella and Shigella infections among under five children with diarrhea was higher than the national prevalence. This study also revealed that the enteric pathogen infection were significantly associated untrimmed finger nail, urban residence, hand washing practice, waste disposal system, usage of napkin and month income of the parent/caregivers. It is also showed that significant amount of the isolates resist commonly used antimicrobial drugs. Therefore, improving hygiene status of under five children and implementation work on identified associated factors with regular drug susceptibility test is important to reduce the problem.

\section{Authors' contributions}

GA participated in the design of the study, conducted data collection, analyzed the data and drafted the paper. TT, EG, and FG participated in the design of the study, analysis of the data and revised subsequent drafts of the paper.

All authors read and approved the final manuscript.

\section{Author details}

${ }^{1}$ Department of Medical Laboratory Science, College of Medicine and Health Sciences, Arba Minch University, P.O. Box: 21, Arba Minch, Ethiopia. ${ }^{2}$ Department of Medical Laboratory Science, Harar Regional Blood Bank, Harar, 
Ethiopia. ${ }^{3}$ Department of Medical Laboratory Science, Yirgachefe primary Hospital, Dilla, Ethiopia.

\section{Acknowledgements}

We are grateful to Arba Minch University, College of Medicine and Health Sciences for supporting this study, we would like to acknowledge Arba Minch town Governmental Health Institutions for permitting as to conduct the study and the laboratory staffs for their assistance during data collection.

\section{Competing interests}

The authors declare that they have no competing interests.

\section{Availability of data and materials}

The datasets supporting the conclusions of this article are included within the article.

\section{Consent for publication}

Written informed consent was obtained from the study participants for publication of this research work during data collection.

\section{Ethics approval and consent to participate}

Ethical clearance was obtained Arba Minch University, College of Medicine and Health Sciences Institutional ethical review board. Official permission letter was given to Arba Minch hospital and Arba Minch town Health Centers before data collection. Information about objective of the study and procedures was given before participant enrolled to the study. Oral assent and written informed consent was obtained from each mother or caregiver of children selected for the study.

\section{Funding}

No funding obtained for this study.

\section{Publisher's Note}

Springer Nature remains neutral with regard to jurisdictional claims in published maps and institutional affiliations.

Received: 20 October 2017 Accepted: 19 January 2018

Published online: 01 February 2018

\section{References}

1. Kosek M, Bern C, Guerrant RL. The global burden of diarrhoeal disease, as estimated from studies published between 1992 and 2000. Bull World Health Organ. 2003;81:197-204.

2. World Health Organization. Diarrheal disease. 2015. http://www.who.int/ mediacentre/factsheets/fs330/en/. Accessed 16 Aug 2017.

3. UNICEF/WHO, diarrhea. Why children are still dying and what can be done. 2009. http://apps.who.int/iris/bitstr eam/10665/44174/1/9789241598415_eng.pdf. Accessed 16 Aug 2017.

4. Vu Nguyen T, Le Van P, Le Huy C, Nguyen Gia K, Weintraub A. Etiology and epidemiology of diarrhea in children in Hanoi, Vietnam. Int J Infect. 2006;10:298-308.

5. Guarino A, Albano F, Ashkenazi S, Gendrel D, Hoekstra JH, Shamir R, et al. European Society for Paediatric Gastroenterology, Hepatology, and Nutrition/European Society for Paediatric Infectious Diseases evidence-based guidelines for the management of acute gastroenteritis in children in Europe: executive summary. J Pediatr Gastroenterol Nutr. 2008:46(5):619-21.

6. Viswanathan K, Hodges K, Hect G. Enteric infection meets intestinal function: how bacterial pathogens cause diarrhea. Nat Rev Microbiol. 2009;7:110-9

7. Hodges K, Gill R. Infectious diarrheal: cellular and molecular mechanisms. Gut Microb. 2010;1(1):4-21.

8. World Health Organization. Typhoid vaccine. Weekly epidemiology. 2008;83(6): 49-60. http://www.who.int/wer/2008/wer8306/en/. Accessed 22 Aug 2017.
9. Clemens J, Kotloff K, Kay B. Generic protocol to estimate the burden of Shigella diarrhea and dysenteric mortality. Geneva: World Health Organization: Department of Vaccines and Biologicals; 1999.

10. Murrey P, Rosenthal K, Pfaller M. Medical microbiology. 7th ed. New York: Elsevier; 2014. p. 264-6.

11. Jamison DT, Breman JG, Measham AR, Alleyne G, Claeson M, Evans DB, et al. Disease control priorities in developing countries. 2nd ed. Washington, DC: World Bank and Oxford University Press; 2006.

12. World Health Organization. Antimicrobial resistance; global report on surveillance. 2014. http://apps.who.int/iris/bitstr eam/10665/112642/1/9789241564748 eng.pdf. Accessed 25 Aug 2017.

13. Mamuye Y, Metaferia G, Birhanu A, Desta K, Fantaw S. Isolation and antibiotic susceptibility patterns of Shigella and Salmonella among under 5 children with acute diarrhoea: a cross-sectional study at selected public health facilities in Addis Ababa, Ethiopia. Clin Microbiol. 2015;4:186.

14. Cheesbrough M. District laboratory practice in tropical countries. 2nd ed. Cambridge: Cambridge University Press; 2006.

15. Clinical and Laboratory Standards Institute. Methods for dilution antimicrobial susceptibility tests for bacteria that grow aerobically approved standard. 18th ed. Wayne: Wayne Publications; 2012.

16. Beyene G, Tasew H. Prevalence of intestinal parasite, Shigella and Salmonella species among diarrheal children in Jimma health center, Southwest Ethiopia. Clin Microbiol Antimicrob. 2014;13:10.

17. Getamesay M, Getenet B, Ahmed Z. Prevalence of Shigella, Salmonella and Campylobacter species and their susceptibility pattern among under five children with diarrhea in Hawassa town, South Ethiopia. Ethiop J Health Sci. 2014;24(2):101-8.

18. Ansari S, Sherchand JB, Parajuli K, Mishra SK, Dahal RK, Shrestha S, et al. Bacterial etiology of acute diarrhea in children under five years of age. J Nepal Health Res Counc. 2012;10(22):218-23.

19. Kara TT, Özdemir H, Kurt F, Güriz H, Çiftçi E, Aysev AD, et al. Prevalence of Salmonella and Shigella spp. and antibiotic resistance status in acute childhood gastroenteritis. J Pediatr Inf. 2015;9:102-7.

20. Paton S, Nicolle L, Mwongera M, Kabiru P, Mirza N, Plummer F, et al. Salmonella and Shigella gastroenteritis at a public teaching hospital in Nairobi, Kenya. Infect Control Hosp Epidemiol. 1991;12(12):710-7.

21. Admassu M, Yemane G, Kibret M, Abera B, Nibret E, Adal M. Prevalence and antibiogram of Shigella and Salmonella spp. from under five children with acute diarrhea in Bahir Dar town, Ethiopia. Ethiop J Health Sci Technol. 2015:8(1):27-35.

22. Gebrekidan A, Dejene TA, Kahsay G, Wasihun AG. Prevalence and antimicrobial susceptibility patterns of Shigella among acute diarrheal outpatients in Mekelle hospital, Northern Ethiopia. BMS Res Notes. 2015;8:611.

23. Okolo MO, Garba DE, Stephen E. Isolation and prevalence of bacteria associated with diarrhea in children visiting hospital in Anyigba. Am J Res Commun. 2013;1(8):121-9.

24. Sebunya K, Mpuchane S, Gashe A. Shigella and Salmonella strains isolated from children under 5 years in Gaborone, Botswana, and their antimicrobial susceptibility patterns. Eur J Trop Med Int Health. 2001;6(1):55-9.

25. Akinnibosun Fl, Nwafor FC. Prevalence of diarrhea and antibiotic susceptibility test in children below 5 years at University of Benin Teaching Hospital, Nigeria. Int Res J Public Environ Health. 2015;2(4):49-55.

26. Karambu S, Matiru V, Kiptoo M, Oundo J. Characterization and factors associated with diarrheal diseases caused by enteric bacterial pathogens among children aged five years and below attending lgembe district Hospital, Kenya. Pan Afr Med J. 2013;16:37.

27. Montazeri N, Goettert D, Achberger EC, Johnson CN, Prinyawiwatkul W, Janes ME. Pathogenic enteric viruses and microbial indicators during secondary treatment of municipal wastewater. Appl Environ Microbiol. 2015;81:6436-45.

28. Santamaría J, Toranzos GA. Enteric pathogens and soil: a short review. Int Microbiol. 2003;6:5-9.

29. Hashi A, Kumie A, Gasana J. Prevalence of diarrhea and associated factors among under-five children in Jigjiga district, Somali region, eastern Ethiopia. Open J Prev Med. 2016;6:233-46. 